\title{
Landmark tool assesses Canadian hospitals
}

$\mathrm{W}$

hile the overall quality, safety and cost-effectiveness of care has improved in Canadian hospitals since 2007, there remain "substantial variations" in performance at the provincial, regional and facility levels, according to a groundbreaking database of clinical and financial data for some 600 institutions.

There were fewer deaths after major surgery, heart attack and stroke; fewer readmissions after heart attack, stroke, and hip and knee surgery; and fewer cases of in-hospital hip fracture in Canadian hospitals in 2010 than in 2007, according to the new Web-based resource released by the Canadian Institute for Health Information (CIHI).

But major anomalies are apparent in the data. For example, in fiscal 2010/11, 30-day in-hospital mortality rates after stroke were higher in Eastern Canada, where the maximum provincial rate was $20.4 \%$, than in Western and Central Canada, where the maximum provincial rate was $15.9 \%$.

The interactive benchmarking tool is aimed at helping hospitals better understand their performance in comparison with their peers (www.cihi.ca /CIHI-ext-portal/internet/en/documentfull /health+system+performance/indicators /performance/indicator_ent). It allows for comparison across 21 clinical indicators and nine financial indicators of hospital effectiveness, governance, patient safety and appropriateness and accessibility, using four years of national, provincial, regional and facility-specific data compiled as part of CIHI's Canadian Hospital Reporting Project.

"As it stands today, it's the most advanced tool of its kind in the world," Jeremy Veillard, vice president for research and analysis at CIHI, asserted during an online technical briefing.

The Web-based resource is the first in Canada to include comprehensive comparative performance data for smaller facilities, which account for about $60 \%$ of hospitals in the country, he added. "Now, we're making some headway in including every hospital in the discussion on performance, benchmarking and peer learning."

The results show that about half of Canada's facilities are performing at or

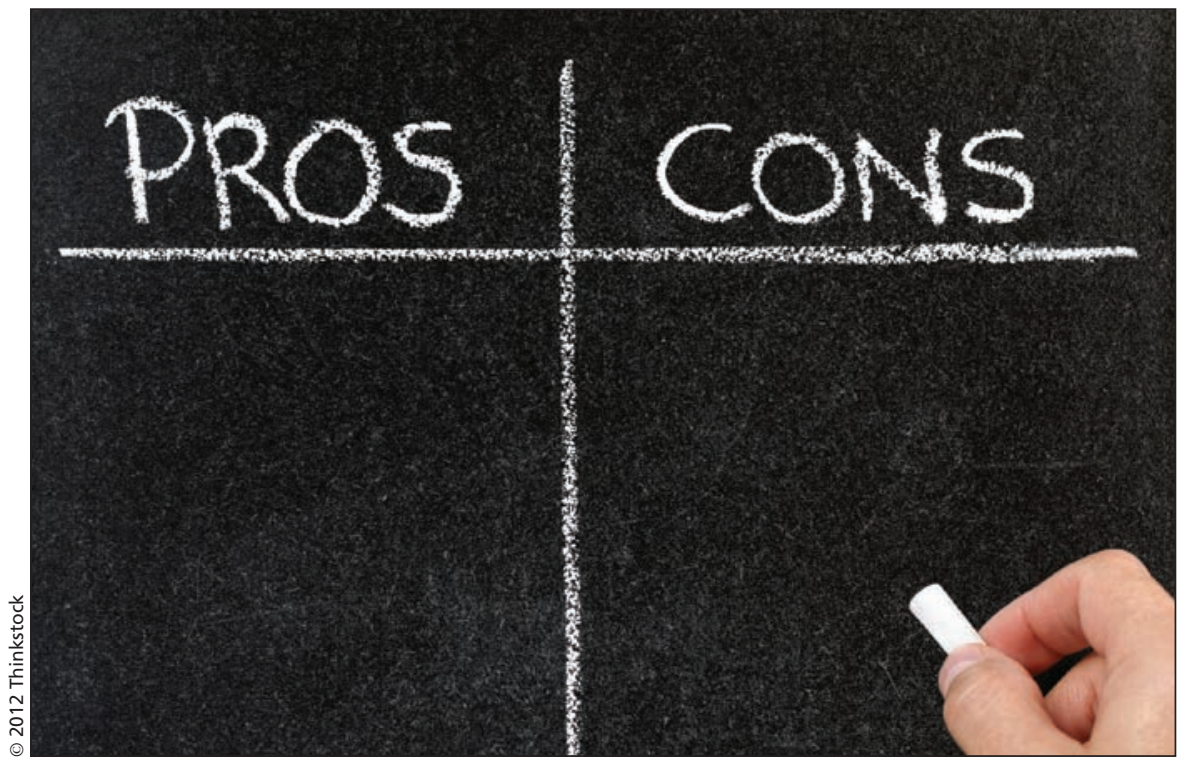

A new Web-based benchmarking tool allows for comparison between hospitals across 21 clinical indicators and 9 financial indicators.

above average on clinical indicators, Kira Leeb, director of health system performance at CIHI, told the technical briefing. Moreover, "we've seen about a $6.7 \%$ decrease in administration costs as a percentage of total costs across hospitals."

Nevertheless, there are still "quite wide variations" between provinces, hospital peer groups and individual facilities, she added.

There was a twofold variation $(4.9 \%$ to $8.2 \%$ ) in provincial rates for 28-day readmission after stroke over the course of one year.

Administration costs also varied substantially by province, Leeb said. In 2009/10, "Ontario had the highest administration costs as a percentage of total costs at $5.9 \%$ and Alberta had the lowest percentage at $3.5 \%$."

There were also major variations by peer group. Large community hospitals, for example, experienced significantly lower-than-average 30-day readmission rates for all patient groups in 2010/11, as well as significantly lower rates of obstetric trauma, as compared with teaching hospitals and smaller community hospitals.

Teaching hospitals, though, comprised the only group with an elective C-section rate $(23.9 \%)$ lower than the Canadian average (25.6\%) in 2009/10.
There were also variations within peer groups. Most dramatically, mortality rates within five days of major surgery at large community hospitals varied from 2.2 per 1000 to 16.5 per 1000 in 2010/11 — an eightfold variation.

Similarly, the 28-day readmission rate after stroke in 2010/11 within teaching hospitals varied threefold (3.8\% to $12.0 \%)$.

"Even within teaching hospitals, for example, you can have slight differences with the types of procedures and volumes they do," says Jeanie Lacroix, manager of hospital reports at CIHI. "Variations can be related to a number of factors at the community level, [such as] how the hospital adheres to clinical practice guidelines, resource availability and effectiveness of treatment in the hospital.'

For that reason, the benchmarking tool includes facility and community profile information for each hospital so users can flesh out that context, she adds.

But ultimately, "no one measure can reflect all aspects of quality of care," Lacroix says. "This is a starting point and we hope to work and develop more indicators so we can look at performance across more areas in the future." — Lauren Vogel, CMAJ

CMAJ 2012. DOI:10.1503/cmaj.109-4178 